\section{Facharztprüfung zur Erlangung des Facharzttitels FMH für Chirurgie}

Aufgrund des Weiterbildungsprogrammes und des Beschlusses des Zentralvorstandes der FMH vom 3. Februar 1999 ist das Bestehen der Facharztprüfung für alle Kandidatinnen und Kandidaten, welche ihre Weiterbildung nach dem 31. Dezember 2000 abschliessen, Voraussetzung zur Erlangung des Facharzttitels für Chirurgie. Näheres zu den Übergangsbestimmungen und Ausnahmen wurde in der Schweizerischen Ärztezeitung Nr. 6 vom 7. Februar 2001 publiziert.

Die Facharztprüfung besteht aus zwei Teilen:

1. Teil: über allgemeine chirurgische Kenntnisse (Basisexamen Chirurgie);

2. Teil: über spezielles chirurgisches Fachwissen.

Der 1. Teil, das Basisexamen Chirurgie, wird von der Schweizerischen Union der Chirurgischen Fachgesellschaften durchgeführt (schriftlich, 4 Std., 150 Fragen [Information: www.surgery.ch]).

Der 2. Teil erfolgt mündlich: die Kandidatin/der Kandidat wird von mehreren Examinatoren anhand von 6 standardisierten Patienten-Fallbeispielen geprüft. Dauer: $3 \times 30$ Minuten.
Es empfiehlt sich, den 1. Teil der Prüfung (Basisexamen) in der Regel nach den ersten zwei Jahren der Weiterbildung in allgemeiner Chirurgie zu absolvieren.

Es empfiehlt sich, den 2. Teil frühestens im letzten Jahr der Weiterbildung zu absolvieren. Zum 2. Teil wird nur zugelassen, wer den 1. Teil mit Erfolg bestanden hat.

\section{Prüfungsdaten}

1. Teil: Samstag, 10. November 2001, Inselspital, Bern und CHUV, Lausanne

2. Teil: Mittwoch bis Freitag, 7. bis 9. November 2001, Inselspital, Bern

Prüfungsgebühr

1. Teil: Fr. 300.-

2. Teil: Fr. 990.-

\section{Anmeldung}

1. Teil: Sekretariat Chirurgische Klinik, Kantonsspital, 9007 St. Gallen, Tel. 0714942943

2. Teil: Sekretariat der Schweizerischen Gesellschaft für Chirurgie, Postfach 1527, 4133 Pratteln, Tel. 06181596 60, E-mail: info@sgc-ssc.ch

\section{Anmeldefrist}

1. Teil: 30. Juni 2001

2. Teil: 31. Juli 2001

\section{Prüfung der Grundkenntnisse in Chirurgie (Basisexamen Chirurgie)}

Für die Chirurgischen Fachgesellschaften, die aufgrund ihres Weiterbildungsprogrammes die Prüfung der allgemeinen Grundkenntnisse (Basisexamen Chirurgie) als Voraussetzung zur Erlangung des Facharzttitels vorsehen, führt die Union der Chirurgischen Fachgesellschaften das Basisexamen Chirurgie durch.

Art der Prüfung: Schriftliche Prüfung nach dem Wahlantwortverfahren mit 150 Fragen in 4 Stunden.

Zeitpunkt: Es empfiehlt sich, das Basisexamen in der Regel nach den ersten zwei Jahren der Weiterbildung in allgemeiner Chirurgie zu absolvieren.
Prüfungsdaten: Samstag, 10. November 2001, Inselspital Bern und CHUV Lausanne

Information: Sämtliche Informationen sowie das Anmeldeformular sind im Internet unter www. surgery.ch abrufbar, eine Broschüre mit Lernzielkatalog, Blueprint und Reglement sowie ein Modellheft erhalten die angemeldeten Kandidat/innen.

Prüfungsgebühr: Fr. 300.-

Anmeldung: Beim Sekretariat Chirurgische Klinik, Kantonsspital, 9007 St. Gallen, Tel. 0714942943. Formulare sind beim Chefarzt Ihrer Klinik, oder beim Sekretariat der Chirurgischen Klinik, Kantonsspital, 9007 St. Gallen erhältlich.

Anmeldefrist: 30. Juni 2001 


\section{Examen de spécialiste en vue de l'obtention du titre de spécialiste FMH en chirurgie}

Conformément au programme de formation postgraduée et à la décision du Comité central de la FMH du 3 février 1999, la réussite de l'examen de spécialiste est une condition requise pour les candidats au titre en chirurgie qui termineront leur formation postgraduée après le 31 décembre 2000. Pour de plus amples renseignements concernant les dispositions transitoires et les dérogations, veuillez vous référer à la publication du Bulletin des médecins suisses $\mathrm{n}^{\circ}$ 6, du 7 février 2001.

L'examen de spécialiste comprend deux parties: $1^{\text {re }}$ partie: chirurgie générale (connaissances de base);

$2^{\mathrm{e}}$ partie: connaissances chirurgicales spécifiques. La $1^{\text {re }}$ partie de l'examen de base est organisée par l'Union suisse des sociétés chirurgicales (examen écrit, 150 QCM en 4 heures [information: www. surgery.ch]).

La $2^{\mathrm{e}}$ partie consiste en un examen oral; le/la candidat/e est interrogé/e par plusieurs examinateurs sur la base de cas types (au nombre de 6). Durée: $3 \times 30$ min.
D’une manière générale, il est conseillé de passer la $1^{\text {re }}$ partie de l'examen (examen de base) après les deux premières années de formation postgraduée en chirurgie générale.

Il est recommandé de passer la $2^{\mathrm{e}}$ partie de l'examen au plus tôt durant la dernière année de formation postgraduée. Chaque candidat doit avoir réussi la $1^{\text {re }}$ partie pour pouvoir se présenter à la deuxième.

\section{Dates d'examen}

$1^{\text {re }}$ partie: le samedi, 10 novembre 2001, à l'Hôpital de l'Ile, Berne et au CHUV, Lausanne

$2^{\mathrm{e}}$ partie: du mercredi au vendredi, 7 au 9 novembre 2001, à l'Hôpital de l'Ile, Berne

\section{Taxe d'examen}

$1^{\text {re }}$ partie: Fr. 300.-

$2^{\mathrm{e}}$ partie: Fr. 990.-

\section{Inscription}

$1^{\text {re }}$ partie: Secrétariat de la Clinique chirurgicale, Hôpital cantonal, 9007 Saint-Gall, tél. 0714942943 $2^{\text {e }}$ partie: Secrétariat de la Société suisse de Chirurgie, Case postale 1527, 4133 Pratteln, tél. 06181596 60, e-mail: info@sgc-ssc.ch

\section{Délai d'inscription}

$1^{\text {re }}$ partie: 30 juin 2001

$2^{\mathrm{e}}$ partie: 31 juillet 2001

\section{Examen des connaissances de base en chirurgie}

L'examen de base en chirurgie est organisé par l'Union suisse des sociétés chirurgicales pour les sociétés où, conformément à leur programme de formation postgraduée, la réussite de l'examen des connaissances de base est une condition requise pour l'obtention du titre de spécialiste FMH.

Type d'examen: Examen écrit avec 150 questions à choix multiple d'une durée de 4 heures.

Moment de l'examen: D'une manière générale, il est recommandé de passer l'examen après les deux premières années de formation postgraduée en chirurgie générale.
Date d'examen: Samedi le 10 novembre 2001 à l'Hôpital de l'Ile à Berne et au CHUV à Lausanne

Information: Tous les renseignements ainsi que les formulaires d'inscription sont disponibles sur internet. www.surgery.ch. Les candidats inscrits reçoivent un carnet comprenant le catalogue des objectifs d'apprentissage, le blueprint, le règlement ainsi qu'un examen modèle.

Taxe d'examen: Fr. 300.-

Inscription: Auprès du Secrétariat de la Clinique chirurgicale, Hôpital cantonal, 9007 St-Gall, tél. 07149429 43. Les formulaires peuvent être obtenus auprès du médecin-chef de votre clinique ou auprès du Secrétariat de la Clinique chirurgicale, Hôpital cantonal, 9007 St-Gall.

Délai d'inscription: 30 juin 2001 